University of Nebraska - Lincoln

DigitalCommons@University of Nebraska - Lincoln

Norman R. Simon Papers

Research Papers in Physics and Astronomy

$1-10-1994$

\title{
COMPARATIVE PULSATION CALCULATIONS WITH OP AND OPAL OPACITIES
}

Shashi M. Kanbur

University of Nebraska-Lincoln, kanbur@oswego.edu

Norman R. Simon

University of Nebraska - Lincoln, nsimon@unl.edu

Follow this and additional works at: https://digitalcommons.unl.edu/physicssimon

Kanbur, Shashi M. and Simon, Norman R., "COMPARATIVE PULSATION CALCULATIONS WITH OP AND

OPAL OPACITIES" (1994). Norman R. Simon Papers. 42.

https://digitalcommons.unl.edu/physicssimon/42

This Article is brought to you for free and open access by the Research Papers in Physics and Astronomy at DigitalCommons@University of Nebraska - Lincoln. It has been accepted for inclusion in Norman R. Simon Papers by an authorized administrator of DigitalCommons@University of Nebraska - Lincoln. 


\author{
(1)
}

\title{
COMPARATIVE PULSATION CALCULATIONS WITH OP AND OPAL OPACITIES
}

\author{
Shashi M. Kanbur and Norman R. Simon \\ Department of Physics and Astronomy, University of Nebraska-Lincoln, Lincoln, NE 68588-0111 \\ Received 1992 May 27; accepted 1993 July 20
}

\begin{abstract}
Comparative linear nonadiabatic pulsation calculations are presented using the OPAL and Opacity Project opacities. The two sets of opacities include effects due to intermediate coupling and fine structure as well as new abundances. We used two mass luminosity $(\mathrm{M}-\mathrm{L})$ relations, one standard (BIT), and one employing substantial convective core overshoot (COV). The two sets of opacities cannot be differentiated on the basis of the stellar pulsation calculations presented here. The BIT relation can model the beat and bump Cepheids with masses between 4 and $7 M_{\odot}$, while if the overshoot relation is used, masses between 2 and $6 M_{\odot}$ are required. In the RR Lyrae regime, we find the inferred masses of globular cluster RRd stars to be little influenced by the choice of OPAL or OP. Finally, the limited modeling we have done is not able to constrain the Cepheid $M-L$ relation based upon period ratios observed in the beat and bump stars.
\end{abstract}

Subject headings: atomic data - Cepheids - stars: interiors - stars: oscillations -

stars: variables: other (RR Lyrae)

\section{INTRODUCTION}

The OPAL opacities (Iglesias \& Rogers 1991; Rogers \& Iglesias 1991; Iglesias, Rogers, \& Wilson 1992) are recent revisions to the traditional Los Alamos opacities and have proven very successful in resolving the Cepheid beat mass, and to a lesser extent, the Cepheid bump mass discrepancies (Moskalik, Buchler, \& Marom 1991, hereafter MBM). MBM showed that for the beat Cepheids the $P 1 / P 0$ period ratios, calculated with OPAL, yielded masses between 4 and $7 M_{\odot}$, in agreement with other mass determinations. However, they reported that use of the OPAL opacities still failed to give stable double mode pulsation. MBM also found that the $P 2 / P 0$ period ratios were reduced, implying higher bump Cepheid masses, although the conflict with standard evolutionary masses was not completely eliminated. In parallel, Kovacs, Bucler, \& Marom (1991, hereafter KBM) showed that RR Lyrae mass determinations using the RRd stars were significantly affected when OPAL opacities were employed in place of the Los Alamos version. It is the purpose of the pesent paper to display the results of linear nonadiabatic (LNA) pulsation calculations with yet another set of recently computed opacities, namely, those of the Opacity Project (Seaton 1987; Seaton et al. 1993).

Opacity calculations require extensive atomic data and an equation of state. The Opacity Project (OP) used ab initio methods to compute the required atomic data, whereas OPAL used parametric potentials. The two teams also employed different approaches for the equations of state. OP used the "chemical" picture, where atoms are perturbed by the surrounding plasma (Hummer \& Mihalas 1988) while OPAL used a "physical" picture considering only electrons and nuclei (Rogers 1986). A comparison of the OPAL and OP opacities shows strong similarities, but it is of interest to compare the pulsation results using the two sets of calculations. Note that the calculations presented here used opacities which included effects due to intermediate coupling (Iglesias, Rogers, \& Wilson 1992) and fine structure (Seaton et al. 1993), and also employed new relative abundances of metals due to Grevesse et al. (1990, 1991).

\section{THE NUMERICAL TREATMENT}

Both OP and OPAL tables have been supplied to us in the form of a rectangular grid with $\log \kappa$ given as a function of $\log$ $T_{6}$ and $\log R$, where $t_{6}=T / 10^{6}$ and $R=\rho /\left(T_{6}\right)^{3}$. A twodimensional cubic spline was used to make an extremely fine mesh of $\log \kappa$ in $\log T_{6}$ and $\log R$. The derivatives of $\log \kappa$ with respect to temperature and density were obtained by numerical differentiation. The LNA code used linear interpolation in this fine table to compute $\kappa$ and its derivatives with respect to temperature and density. The procedure was checked by comparing our results for OPAL with those obtained by MBM and KBM. Close agreement was obtained for periods, period ratios and growth rates. In addition, the results were stable to changes in such factors as zoning and interior and exterior boundary conditions.

The pulsation calculations were performed using the LNA code described in Aikawa \& Simon (1983). The static models were constructed with $\sim 70-80$ mass zones, including fine zoning in the hydrogen ionization region. The models were completely radiative and were integrated to an inferior fractional radius of 0.1 for Cepheids and 0.05 for RR Lyraes.

\section{BEAT CEPHEIDS}

Beat Cepheids pulsate simultaneously in the fundamental and first overtone modes. The periods and period ratios of these double-mode stars have been measured very accurately. Initially, in our study, we follow the approach of MBM who constructed model sequences according to the Becker, Iben, \& Tuggle (1977, hereafter BIT) mass luminosity $(M-L)$ relation. However, we have used the recent revisions to the OPAL tables (Iglesias et al. 1992), which accounted for effects due to intermediate coupling and also new abundances from Grevesse et al. $(1990,1991)$. Our results in the Petersen diagram in Figure 1 are a confirmation of the work of MBM and are given here for comparison with our results using the OP opacities. In this and in Figure 2, each line is a one-parameter family with the same mass, luminosity, and composition, but variable effective temperature. The mass used (in solar masses) is indicated next to each line. The luminosity used for each mass came 


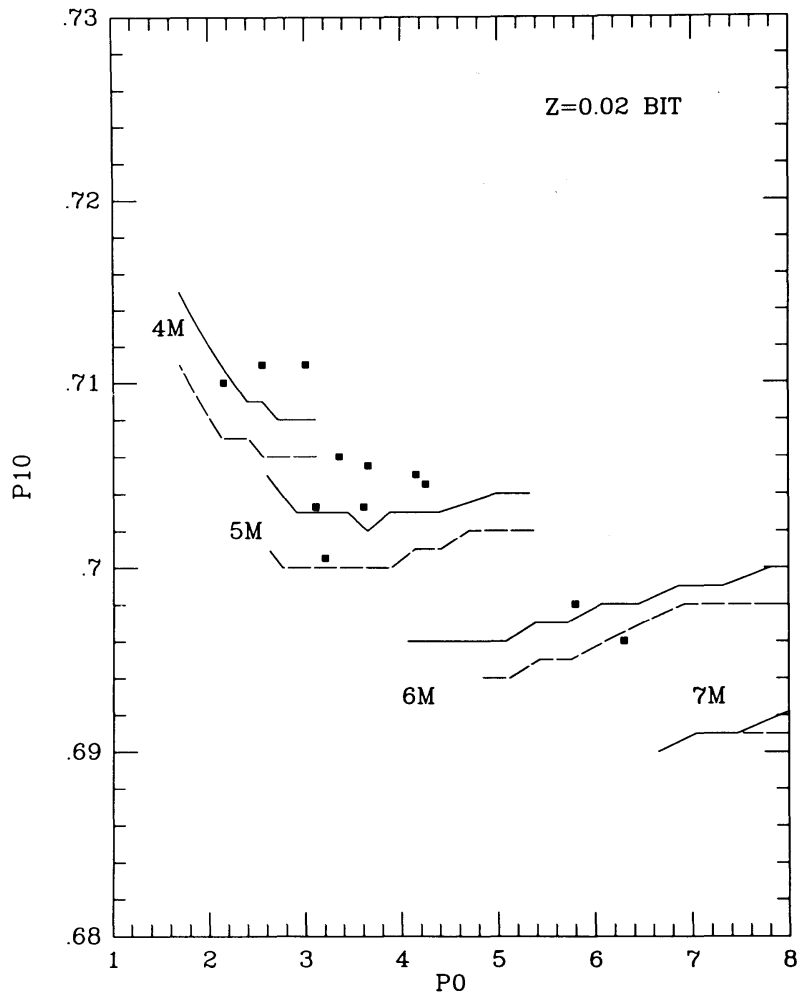

Fig. 1. $-P_{1} / P_{0}$ ratios against $P_{0}(d)$ with $Z=0.02$ and BIT. Solid and dashed lines: LNA models with OPAL and OP, respectively (masses are indicated). Filled squares are observed beat Cepheids.

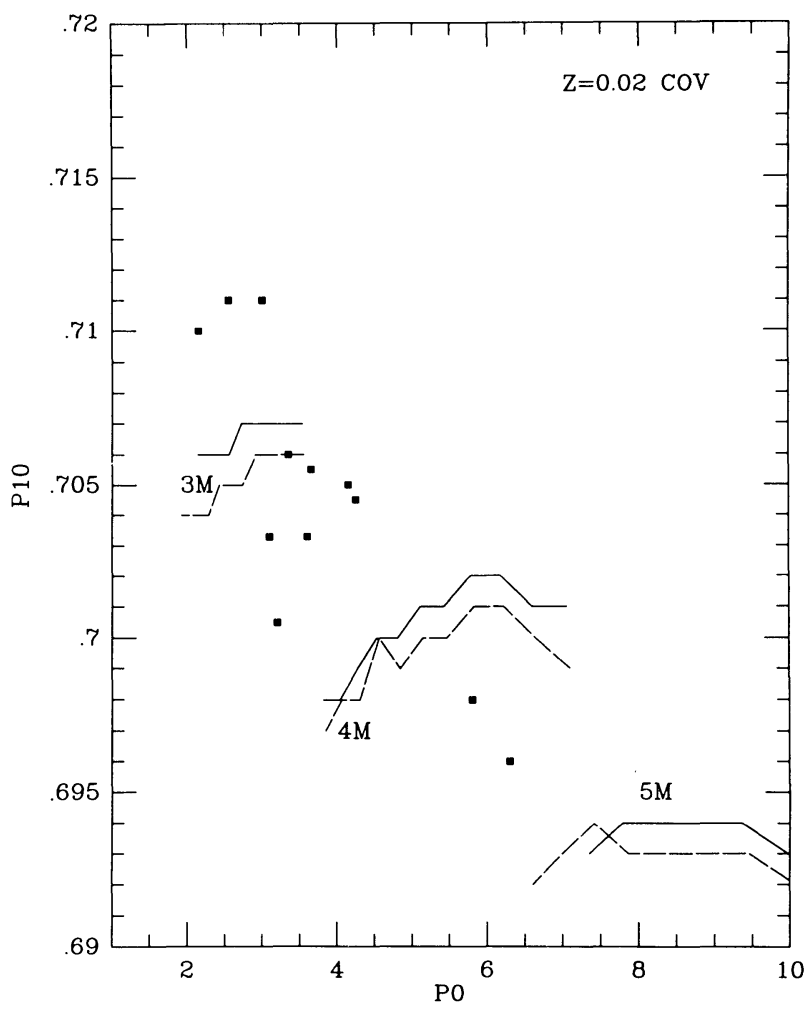

FIG. 2.-Same as Fig. 1, except that $\mathrm{COV}$ is used. either from BIT,

$$
\log \left(L / L_{\odot}\right)=0.46+3.68 \log \left(M / M_{\odot}\right),
$$

or from the $M-L$ relation obtained from models with substantial core overshoot (see, e.g., Chiosi 1989),

$$
\log \left(L / L_{\odot}\right)=0.924+3.61 \log \left(M / M_{\odot}\right),
$$

hereafter designated by COV. These are indicated on the figures. Solid lines refer to our calculations with OPAL, and dashed lines refer to OP calculations. At the short-period end, the lines terminate at an effective temperature close to the fundamental blue edge. The vertical axis is the beat period ratio, $P_{1} / P_{0}$, and the horizontal axis is the fundamental period, $P_{0}$, in days. The metallicity is $Z=0.02$. Solid squares refer to observations of the dozen known double mode Cepheids (MBM and references therein).

Figure 1 shows that both sets of new opacities reduce the period ratios considerably compared with Los Alamos opacities (see MBM), and both the OP and OPAL period ratios coincide crudely with the locus of observed points. As is the case with OPAL, the OP period ratios are systematically less than OPAL, but the difference is small, of the order of 0.005 in $P_{1} / P_{0}$. Figure 2 shows a similar set of calculations using the COV $M-L$ relation. Again, both sets of opacities produce similar period ratios, although with $\mathrm{COV}$ a given period ratio will be obtained with a smaller mass than that required by BIT. Hence COV and BIT need masses in the range $2-5 M_{\odot}$ and 4 to $7 M_{\odot}$, respectively, to crudely match the observed doublemode Cepheids.

\section{BUMP CEPHEIDS}

Bump Cepheids show a discernable progression of light curve shape with period (Hertzsprung 1926). The work of Simon \& Schmidt (1976), Simon \& Lee (1981) and Buchler, Moskalik, \& Kovacs (1990) showed that the progression is related to a 2:1 resonance between the fundamental and second overtone such that $P_{2} / P_{0}=0.5$ with the resonance center near $P_{0}=10 \mathrm{~d}$. The historical problem was that models calculated with the Los Alamos opacities and constrained by standard $M-L$ relations gave $P_{2} / P_{0}=0.5$ at a fundamental period much larger than 10 days. Thus the period ratios were too high, or equivalently, masses $\sim 30-40 \%$ lower than evolutionary masses were required.

MBM showed that the OPAL opacities considerably increased the implied bump Cepheid masses. They adopted a luminosity of $3865 L_{\odot}$ given by Gieren (1988) and looked at the $P_{2} / P_{0}$ ratio for a range of masses and temperatures. They found that a model of $5 M_{\odot}$ came close to satisfying the resonance condition. However, for this mass, the BIT and COV $M-L$ relations imply luminosities of 1077 and $2800 L_{\odot}$, respectively. Since the same $M-L$ relations should presumably apply in both the beat and bump Cepheid cases, we have departed from MBM and used the BIT and COV relations in our bump Cepheid models. Our results are displayed in Figures 3 and 4 . These figures are set out similarly to Figures 1 and 2 , except that the vertical axis now refers to the bump period ratio $P_{2} / P_{0}$. The cross in the figures marks the approximate position of the resonance $\left(P_{2} / P_{0}=0.5\right.$ at $\left.P_{0}=10^{d}\right)$. Comparing the OPAL and OP period ratios, one again notes that the latter tend to be slightly smaller, by an amount of less than 0.005 . 


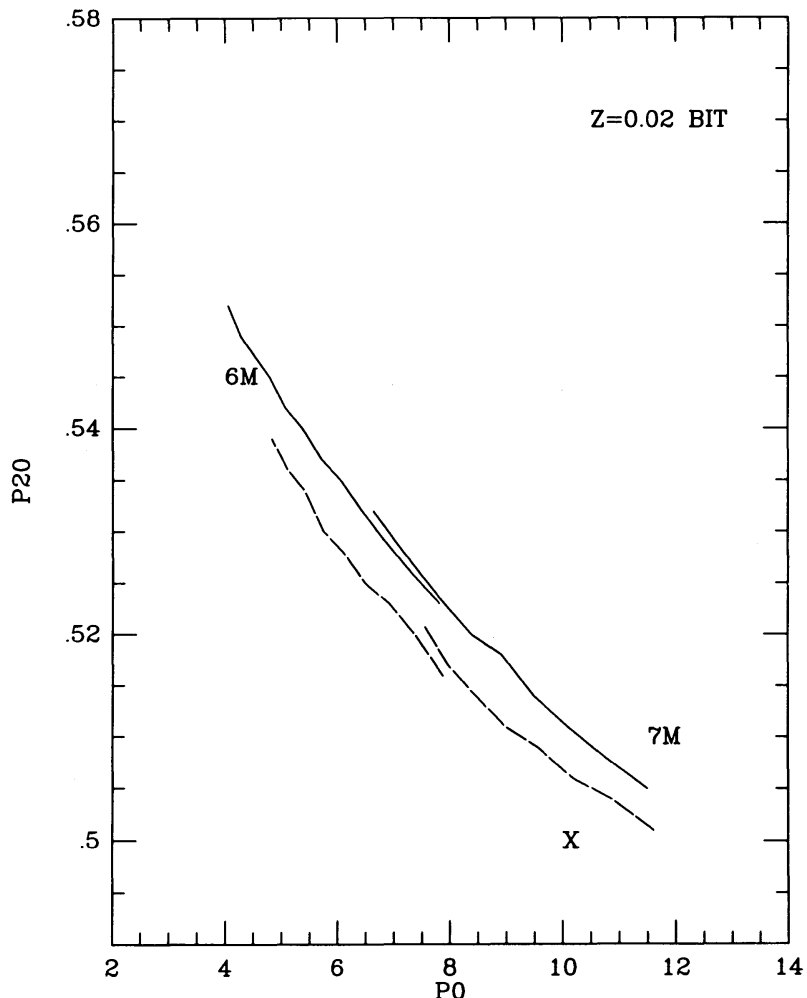

Fig. 3. $-P_{2} / P_{0}$ ratios against $P_{0}(d)$ with $Z=0.02$ and BIT. Solid lines are OPAL, dashed lines are OP (masses are indicated). Cross marks resonance position.

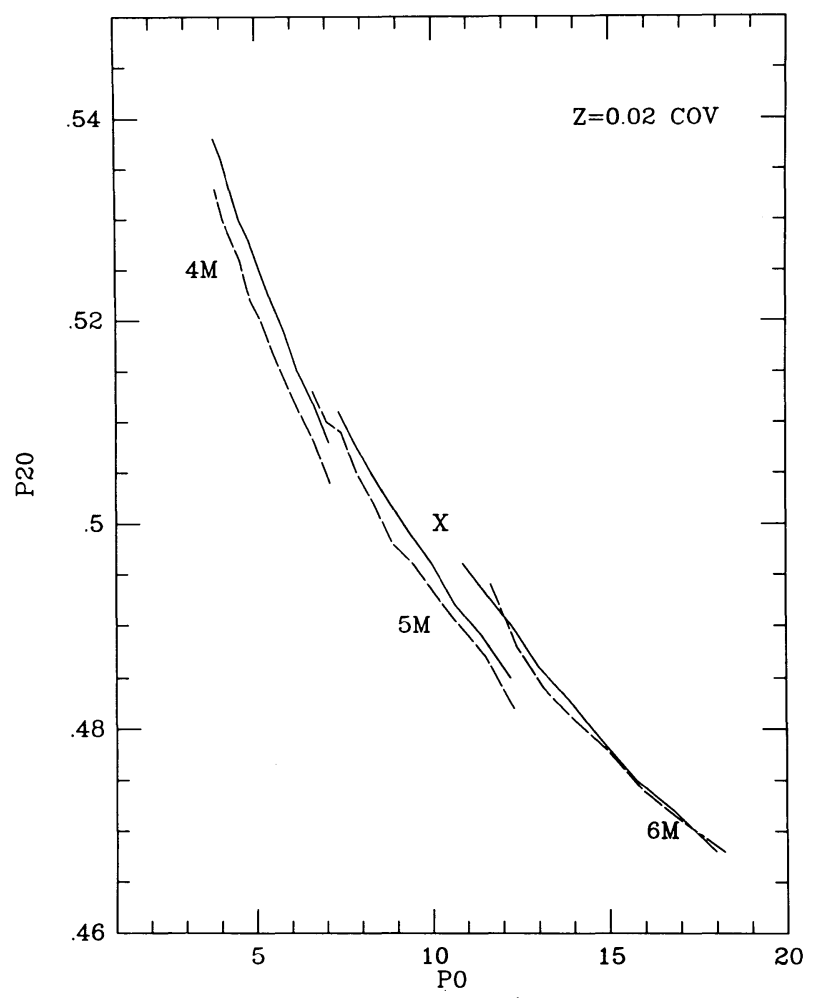

FIG. 4.-Same as Fig. 3, except that $\mathrm{COV}$ is used
Figure 3 shows that for the BIT relation, OP comes closest to matching the resonance condition with a period ratio of $\sim 0.505$ at a period of 10.3. This particular model had $M=$ $6.5 M_{\odot}$ and $T_{e}=5000 \mathrm{~K}$. OPAL yields a period ratio of 0.51 at a similar period. With the COV relation, Figure 4 shows that both OPAL and OP come close to achieving the resonance condition with masses around $5 M_{\odot}$. In fact a $5.5 M_{\odot}$ model yeilds a period ratio of 0.496 at a fundamental period of 9.95 .

\section{RRd STARS}

The RRd stars are RR Lyraes which pulsate simultaneously in the first overtone and fundamental modes. Obtaining their masses is important in understanding stellar evolution and the history of the Galaxy. They have been found in both Oosterhoff I (Oo I) and Oosterhoff II (Oo II) globular clusters. The Oo I RRd clusters have metallicities of the order $Z=0.0005$, and RRd stars with average periods and period ratios given by $P_{0}=0$ d $48, P_{1} / P_{0}=0.7444(\mathrm{KBM})$. The Oo II clusters are more metal poor $(Z \approx 0.0001)$ with average $R R d$ periods and period ratios given by $P_{0}=0.55, P_{1} / P_{0}=0.7460(\mathrm{KBM})$. With the Los Alamos opacities, these observed parameters yield masses near $0.55 M_{\odot}$ and $0.65 M_{\odot}$ for the RRd stars in Oo I and Oo II clusters, respectively (Cox 1988; Simon \& Cox 1991). KBM reexamined this problem with the OPAL opacities, and inferred masses of $\sim 0.77 M_{\odot}$ in both IC 4499 (Oo I) and M15 (Oo II). However, this result is highly sensitive to the assumed chemical abundances (Kovacs et al. 1992).

We have calculated RRd models using OP and compared the period ratios, $P_{1} / P_{0}$, with those obtained using OPAL. At $Z=0.0001$, there is virtually no difference. At $Z=0.001$, the OP period ratios are slightly smaller, implying RRd masses which are larger by a relatively small amount $\left(<0.05 M_{\odot}\right)$. Thus for the globular clusters containing RRd stars $(Z \approx 0.0001$ for Oo II and $Z \approx 0.0005$ for Oo I), we expect that the substitution of OP for OPAL would produce little if any effect on the RRd masses.

\section{DISCUSSION}

It is clear from Figures 1-4 that our present calculations cannot distinguish between the OP and OPAL opacities in terms of stellar pulsation results. It is reassuring that two such different approaches to the calculation of radiative opacities can produce very similar results for the beat and bump Cepheid cases. Recently, Stothers \& Chin (1991) have recalculated evolutionary tracks with OPAL and omitting core overshoot. The $M-L$ relation obtained is rather close to that of BIT. To our knowledge, tracks which use OPAL with core overshoot have not yet been published.

Can the pulsation models we have presented be used to constrain stellar evolutionary calculations? Figures 1 and 2 show that to fit the shortest period double mode Cepheids requires masses of $4 M_{\odot}$ and $<3 M_{\odot}$ for BIT and $\mathrm{COV}$, respectively. According to published evolutionary tracks (BIT; Bertelli, Bressan, \& Chiosi 1985), the blue loops for these masses do not penetrate the instability strip. While the problem may be less serious than it first appears (see, e.g., Stothers \& Chin 1991), it is still questionable whether Cepheid masses can really be as low as required above, particularly in the COV case.

When one turns to the bump Cepheid problem both $M-L$ relations come close to the resonance condition $\left(P_{2} / P_{0}=0.5\right.$ at $P_{0}=10$ days), e.g., with $P_{2} / P_{0}=0.505$ at $P_{0}=10^{\mathrm{d} 3}$ for 
$6.5 M_{\odot}$ and $T_{e}=5000 \mathrm{~K}$ for BIT, and $P_{2} / P_{0}=0.498$ at $P_{0}=9.95$ for $5.5 M_{\odot}$ and $T_{e}=5700 \mathrm{~K}$ for COV. While this result seems to mildly favor $\mathrm{COV}$, we cannot say that it is $\rightarrow$ I significant. The uncertainties in the LNA period ratios are simply too large to allow a conclusion on the basis of the limited modeling we have performed. Further progress in the use of the period ratios to test evolutionary tracks will require a substantially greater effort in both the beat and bump Cepheid regimes.

We wish to thank F. Rogers, C. Iglesias, and M. Seaton for making opacities available to us before publication. One of us (N. R. S.) is pleased to acknowledge support under the NASA Astrophysics Theory Program, grant NAGW-2395.
Aikawa, T., \& Simon, N. R. 1983, ApJ, 273, 346

Becker, S. A., Iben, I., \& Tuggle, R. S. 1977, ApJ, 218, 633 (BIT)

Bertelli, G., Bressan, A., \& Chiosi, C. 1985, A\&A, 130, 279

Buchler, J. R., Moskalik, P., \& Kovacs, G. 1990, ApJ, 351, 617

Chiosi, C. 1989, in The Use of Pulsating Stars in Fundamental Problems of Astronomy, ed. E. G. Schmidt (Cambridge University Press), 19

Cox, A. N. 1988, in IAU Colloq. 95, Proc. 2d Conf. on Faint Blue Stars, ed. A. G. Davis Phillip, D. S. Hayes, \& J. W. Liebert (Schenectady:Davis), 161 Gieren, W. P. 1988, ApJ, 329, 790

Grevesse, N., Lambert, D. L., Sauval, A. J., van Dishoeck, E. F., Farmer, C. B., \& Naton, R. H. 1990, A\&A, 232, 225 .1991, A\&A, 242, 488

Hertzsprung, E. 1926, Bull. Astr. Inst. Netherlands, 3, 115

Hummer, D. G., \& Mihalas, D. M. 1988, ApJ, 331, 794

\section{REFERENCES}

Iglesias, C. A., \& Rogers, F. J. 1991, ApJ, 371, 408

Iglesias, C. A., Rogers, F. J., \& Wilson, B. 1992, ApJ, 397, 717

Kovacs, G., Buchler, J. R., \& Marom, A. 1991, A\&A (Letters), 252, L27 (KBM)

Kovacs, G., Buchler, J. R., Marom, A., Iglesias, C. A., \& Rogers, F. J. 1992, A\&A (Letters), 259, L46

Moskalik, P., Buchler, J. R., \& Marom, A. 1992, ApJ, 385, 685 (MBM)

Rogers, F. J. 1986, ApJ, 310, 723

Rogers, F. J., \& Iglesias, C. A. 1992, ApJS, 79, 507

Seaton, M. J. 1987, J. Phys. B., 20, 6363

Seaton, M. J., Yu Yan, Mihals, D., \& Pradhan, A. K. 1993, preprint

Simon, N. R., \& Cox, A. N. 1991, ApJ, 376, 717

Simon, N. R., \& Lee, A. S. 1981, ApJ, 248, 291

Simon, N. R., \& Schmidt, E. G. 1976, ApJ, 205, 162

Stothers, R. B., \& Chin, C. 1991, ApJ, 381, L67 